\title{
Emergency management and an emergency plan for the Gran Sasso National Laboratories: underground laboratories and motorway tunnels
}

\author{
A. Giampaoli, R. Perruzza, M. Tobia \& R. Tartaglia \\ Prevention and Protection Service Department, \\ National Institute of Nuclear Physics, \\ Gran Sasso National Laboratory, Italy
}

\begin{abstract}
The Gran Sasso National Laboratories (LNGS) of the National Institute of Nuclear Physics (INFN) are located in Assergi, at about $120 \mathrm{~km}$ Eastward from Rome. The underground facility are located in the Gran Sasso Massive, under a rock layer of $1400 \mathrm{~m}$ thickness, under the Monte Aquila Peak in the area of the National Park of Gran Sasso and Monti della Laga. The underground laboratories are part of the Gran Sasso complex system, which comprehends also two highway tunnels managed by the "Strada dei Parchi S.p.A." company and a huge water reservoir in the Gran Sasso massive, which supplies drinkable water both to L'Aquila and Teramo counties. Moreover, since 2002, the LNGS are subjected to the European Directive 96/82/EC, because of the storage and use of substances classified as dangerous for the environment according to the European Directive 2003/105/EC.

Local Authorities have studied, discussed, agreed and realized a General Emergency Plan (PEE), which takes into account the possible accident scenarios and describes the preventive and protective actions required to avoid and/or limit the harmful effects resulting from major accidents. The object of the present work is the analysis of an emergency drill figuring the implementation of the PEE of LNGS. These tests are important for screening and evaluating the complex dynamics in terms of organization, infrastructure and psychology that determine the validity of an emergency plan and the guidelines to be followed in emergency management. The drill, entitled "Gran Sasso 2008",
\end{abstract}


simulated a crash between a bus and a car close to the exit of the Underground Laboratories: the crash caused too an important fire inside the Gran Sasso highway tunnel. A great number of institutions have been involved in the drill: Strada dei Parchi S.p.A. Company, Traffic Police, Police, Fire Department, 118, Civil Protection, Prefectures of L'Aquila and Teramo and LNGS, of course. The results have confirmed the effectiveness of the current plan, while emphasizing the ability to increase its efficiency by improving logistical aspects, such as the optimization of emergency vehicles available, and above all the communication among the institutions and entities involved.

Keywords: safety, emergency drill, emergency plan, confined space, motorway tunnel, underground laboratory.

\section{Gran Sasso National Laboratories - INFN}

The Gran Sasso National Laboratories (LNGS) are one of the experimental research centres belonging to the Italian National Institute of Nuclear Physics (INFN). The LNGS facility is made up of two main areas:

- External Operations Centre in Assergi, L'Aquila;

- Underground Laboratories.

Both of the areas are located in the heart of Gran Sasso and Monti della Laga National Park. The underground laboratories, housing about 20 experiments, are located under a rock layer of about $1400 \mathrm{~m}$ thickness, acting as a shield against cosmic radiation; the underground cavity is just in the middle of a huge reservoir, along the Gran Sasso highway tunnels (a double-tunnel 10,500 m long gallery). The research areas in which the laboratories operate or plan to operate, are:

- the study of rare nuclear phenomena;

- the study of more penetrating components of cosmic rays;

- neutrino physics;

- dark matter.

LNGS consist of three experimental halls of about $100 \times 20 \times 20 \mathrm{~m}^{3}$ each: Hall A, Hall B, Hall C. The connection among the halls is achieved by other smaller galleries: Car tunnel, Truck tunnel, connecting tunnels.
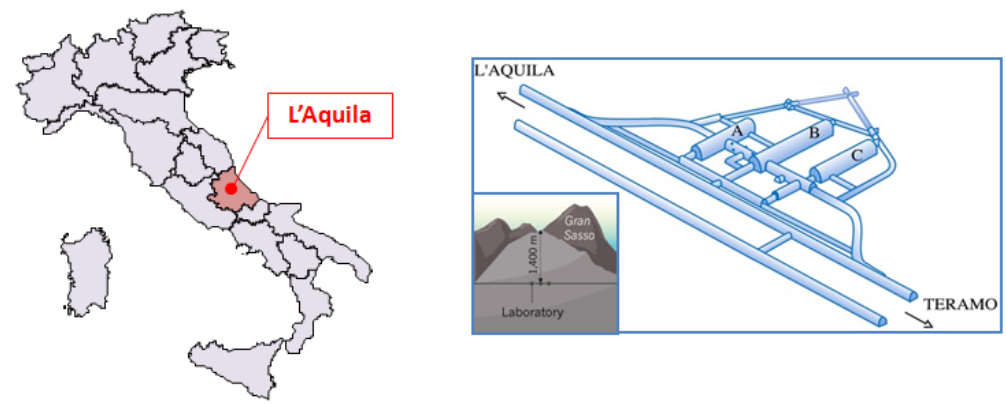

Figure 1: Gran Sasso National Laboratories: Underground Laboratories. 
According to the European Directive Seveso III (2003/105/CE), LNGS are classified as major accident hazard plant because of Experiments using and storing about big amount of substances classified dangerous to the environment [1]. In compliance with Seveso, LNGS have adopted a Safety Management System implemented through 16 Procedures and Operating Instructions. A central element required by the regulation is the clear definition and implementation of an Internal Emergency Plan (PEI). This plan contains all the information, safety measures, procedures and resources needed to ensure the limitation of the consequences of a major accident and, in general, to define how to manage an emergency situation [2]. Furthermore, a General Emergency Plan (PEE) has been agreed and approved, under the co-ordination of L'Aquila Prefecture: the PEE involves Teramo Prefecture, the Company managing the motorway (Strada dei Parchi S.p.A.), the tunnel and labs Company owner (ANAS) L'Aquila and Teramo Fire Brigades, the Police of the Operating Motorway Centre (COA) and the Gran Sasso Laboratories [3].

\section{Gran Sasso tunnel: infrastructure and safety systems}

Gran Sasso tunnels are managed by the Strada dei Parchi Spa company. As regards the infrastructure, the Gran Sasso tunnel is a little bit different from the Mont Blanc one: in fact, it has been built with two "motorway tunnels" interconnected by 18 by-passes [4].

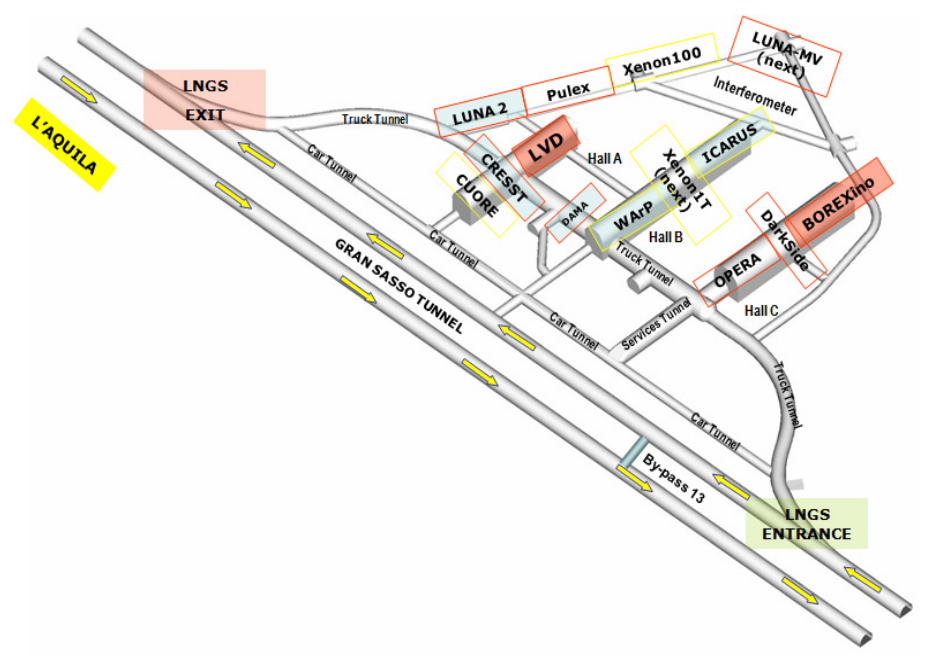

Figure 2: $3 \mathrm{D}$ sketch of Gran Sasso tunnels in the lab area.

The tunnels are not running perfectly parallel and by-passes have different lengths: from $36 \mathrm{~m}$ to $98 \mathrm{~m}$. In case of any event, the by-passes are the real emergency pathway, as the tunnel not involved in the event constitutes "the safe 
place" and the real escape route for the involved people. The tunnels are also equipped with safety systems directly managed by the motorway company. We'd like to point out:

- the ventilation system: gives the possibility to send air at about $40 \mathrm{~km} / \mathrm{h}$. Air flows in the same direction of the traffic and it is not possible to reverse the air flow;

- a thermo-sensing cable is installed in the highest point of the tunnel, with an alarm level set at $70^{\circ} \mathrm{C}$;

- a closed circuit video system controlled by Strada dei Parchi S.p.A.; in particular, there is one TV camera each 200 metres;

- 33 emergency bays, one each 300 metres, in both the tunnels. Each bay is equipped with a fire extinguisher and intercom;

- $\quad$ n.7 ventilation cabins and local transformers at 20.000/380 V.

Moreover, the tunnels are also equipped with safety systems managed by LNGS:

- Terrestrial Trunked Radio (TETRA) communication system, in order to face the absence of a GPS coverage and to be used in case of emergency;

- power supply redundant system in the tunnel, with source both sides - Teramo and L'Aquila;

- redundant system for data connection (phone and ethernet) in the tunnel (transmission both from Teramo and L'Aquila).

ADR Transportation is forbidden in the tunnel: when needed for experimental purposes, the trucks are escorted by police.

For what concerns emergency, Strada dei Parchi S.p.A. has a proper Emergency Assistance able to intervene rapidly in case of accident in the motorway. This Emergency Assistance is equipped with fire-fighting Smart cars, fire-fighting pick-up, fire-fighting scooter. The team is made up of Firemen equipped with High Pressure Water Mist guns. The emergency crew is continuously patrolling the tunnel and/or staying in Assergi, in the operating control room attended and managed by Strada dei Parchi S.p.A. personnel.

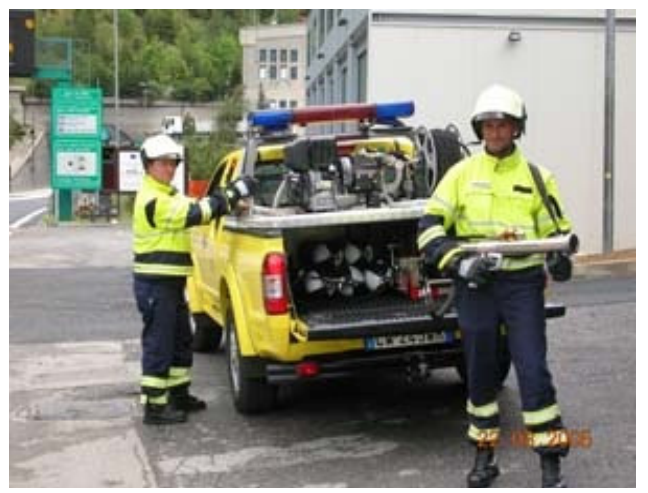

Figure 3: Strada dei Parchi's emergency assistance: firemen with high pressure water mist gun and fire pick-up. 


\section{Gran Sasso general emergency plan: emergency drill organisation}

As previously highlighted, LNGS are part of a very complex system both as regards the safety condition and the environmental constraint such as the logistic point of view. The underground laboratories are located mid-way on the left motorway tunnel, direction Teramo-L'Aquila. The tunnel, roughly $10.5 \mathrm{~km}$ long, connects the Teramo and L'Aquila Counties; the underground labs are at about $6 \mathrm{~km}$ from the L'Aquila side and $5 \mathrm{~km}$ from the Teramo side. The underground LNGS have three areas, confined by fire-proof doors, directly in contact with the motorway: entrance area, exit area and central by-pass.

Before 1993 the tunnel adjacent to the Underground Laboratories was closed to public traffic. The previous configuration, in fact, foresaw the exclusive transit of LNGS' means and employees inside the tunnel. The opening to the public traffic of the TE-AQ tunnel in July 1993, completely changed this configuration. The actual use of the tunnels underlines the need of great coordination efforts between the entities involved, in order to develop an efficient management of ordinary and emergency situations. The close and evident interconnection between the Gran Sasso tunnel and the underground labs is such that any event in the tunnel might cause some effects in the underground labs and vice versa, both as regards the evacuation procedures and as regards the rescue and first aid intervention. Moreover, the Gran Sasso massive is a huge water "reservoir": it is really rich with water and the aqueducts on both the sides (L'Aquila and Teramo) take water beneath the motorway road level in order to guarantee drinkable water supply to both the counties.

All these elements constitute an integral part of the organisational process of the Emergency drill, process which took about 9 months. All the interested institution were involved in periodic meetings to better coordinate all the action foreseen in the drill. The Prefecture of L'Aquila, and, in sub-order, the Teramo one coordinated the meetings among between:

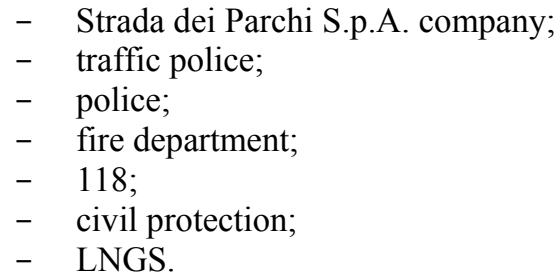

In the months preceding the emergency drill, the meetings have allowed the more detailed definition of the aspects regarding the emergency scenario and the resources involved. Moreover, there was the opportunity to better define the critical parameters and the technical and human factors to be monitored during the drill. 


\section{Gran Sasso 2008: emergency drill}

In order to train the personnel involved in the first aid and rescue and with the goal to verify the efficiency of the general emergency plan, a crew drill, called "Gran Sasso 2008" has been organized and performed on the 14th of May, 2008. The training test involved about 200 people and 30 "rescue vehicles"; moreover, also an intervention of a first-aid 118 helicopter to deliver injured people to the L'Aquila Hospital has been organized. All the entities directly involved into the general emergency plan took part to the crew drill, including also Civil Protection, 118, ASL, Red Cross.

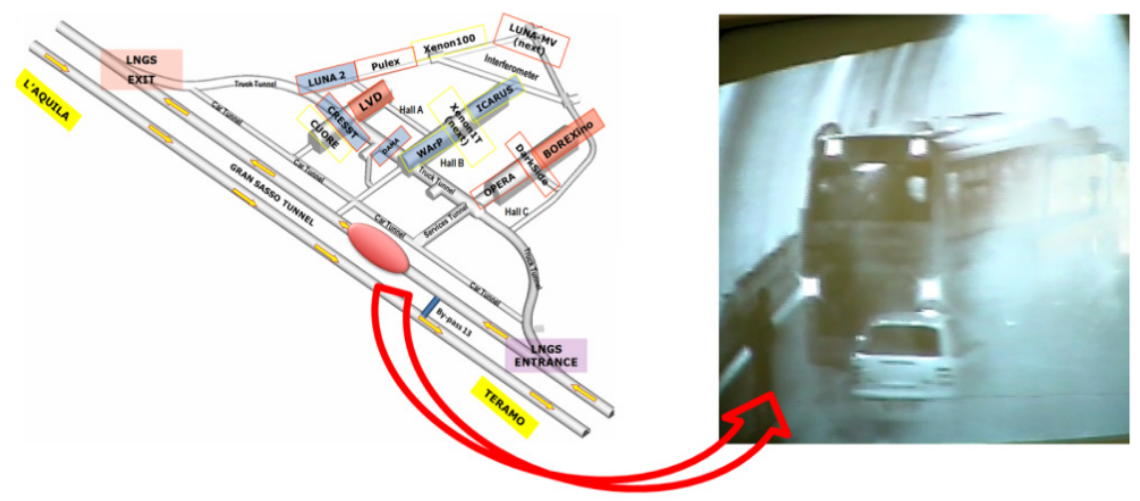

Figure 4: On the left, the position of the simulated accident; on the right reconstruction of the event in the tunnel.

The simulated event has been a crash accident between a bus and a car in an area of the left tunnel between the underground labs central by-pass and the laboratories exit on the L'Aquila side: the crash caused some injured people and a big fire in the tunnel.

At 10:00 a.m. the following operation were performed:

- motorway tunnel closing to the public traffic, in order to guarantee the preparation of the accident scenario;

- bus and car positioning in the crashing place.

With a special device the production of a big amount of non-toxic cold smoke started, filling completely the whole left tunnel. In the Gran Sasso motorway Control Room (Strada dei Parchi S.p.A.) the general command and coordination Centre for all the operation and for the whole crew-drill arrived and gathered. A considerable amount of people took position as "observers" in the pre-defined sites. In order to guarantee to all the observers to follow the entire training test, a video monitoring was available close to the Gran Sasso Control Room. The volunteers who acted as injured people reached their position inside the bus and/or the car. 
At this point the reconstructed accident scenario is the following:

- presence of numerous injured people, some of them really "imprisoned" inside the bus;

- $\quad$ the left tunnel (Direction Teramo-L'Aquila) filled with smoke and heat;

- motorway electrical, communication and vent plants out of service;

- $\quad$ serious damages both to the Laboratories vent and lightening plants;

- urgent need to close both the motorway tunnels to the public traffic and evacuate the lab personnel.

Once completed all the preparing phase, at 0:00 time the crew drill started.

As foreseen in the general emergency plan, the motorway (Strada dei Parchi S.p.A.) operator, once noticed the accident, gave the alarm, communicating the event news to all the entities involved (as reported in the plan) and called the underground labs control room.

The LNGS operator, once alerted through the "red phone", informed the fire fighting crew and the guards on shift underground. Then, thanks to the LNGS operator gave the order to evacuate the labs through the loud-speakers and the acoustic system, inviting all the users present underground to gather in the main assembly point (assembly point number 1), close to the entrance of the labs (LNGS control room). The LNGS fire fighting crew immediately acted as foreseen in the LNGS Emergency plan; they:

* closed the external fire-proof doors, which constitute the real confinement between the underground labs and the motorway tunnel;

* switched the so-called NIAGARA system; it's the system which sprays water on the fire-proof doors themselves, in order to protect and increase the resistance to fire;

* switched the confinement overpressure system on: this system avoids the possible smoke propagation both from the motorway tunnel towards the underground labs and also into the different areas of the underground labs.

Inside the labs, both the guards and firemen wore on the needed PPE - Personnel Protecting Equipment (breathers) and helped researchers, technicians and users to reach as soon as possible the main assembly point, following the light lines and the warning signs. At the same time, all the users received an Oxybox Mask (closed loop breather) to be used in case of emergency. Once gathered all the users in the main assembly point, the guards proceeded with the people counting, in order to verify that everybody reached the assembly point. Then, all the people were staying there, with the Oxybox ready, waiting for instruction by the Fire Brigades.

In the meanwhile, outside the underground laboratories, the first fire brigades vehicles immediately departed from their command centres in L'Aquila and Teramo once alerted. The L'Aquila Fire Brigades, alerted from the motorway command centre at time 0:00, due to the importance and entity of the event, communicated the event to all the other entities involved in the crew drill. During the trip from L'Aquila towards the Gran Sasso tunnel, the operating fire brigades team acquired all the info needed about the event and planned the procedures for intervention. The L'Aquila Fire Brigades run all the right tunnel up to the exit on the Teramo side, then made a U-turn and entered the left 
tunnel, approaching as much as possible the event place. There, they wore on the Personnel Protective Equipment (PPE - breathers) and safely approached as close as possible to the vehicles involved in the accident. At that time the tunnel was completely filled with smoke from the event place up to the tunnel exit on the L'Aquila side. Meanwhile, also the Teramo Fire Brigades reached the event place and, together with the L'Aquila colleagues, proceeded to secure the accident area and devoting themselves to the first aid to the injured people. Once authorized by the Fire Brigades, 2 crews of the 118 entered the tunnel in order to reach the accident area. One 118 doctor and a nurse equipped with breathers (personnel properly trained for the use of these PPE), were taken by the Fire Brigades up to the event place and performed the so-called TRIAGE, assigning a gravity and an evacuation priority code to each injured people. At the same time, the other 118 crew rapidly prepared and organized a First Aid Centre to gather injured and unharmed people inside the by-pass number 13: the Fire Brigades took the injured people and the unharmed ones up to this First Aid Centre, where the ambulances could pick them up and take them to the so-called "Advanced Medical Centres" (AMC). The 118 Doctors, assisted by two professional nurses, performed the triage procedure, assigning the gravity and priority code to the injured people delivered there by the Fire Brigades.

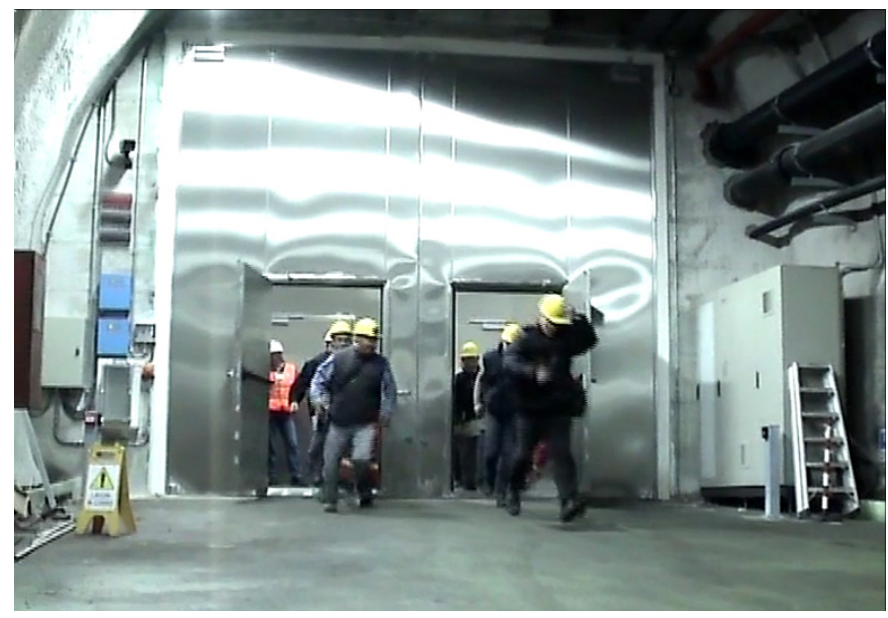

Figure 5: Underground laboratories' users gathering in the main assembly point $n .1$ ready for the people counting by the guards.

Moreover, two AMC were established at the motorway tunnel exits, both on L'Aquila and Teramo side. In each AMC, a team co-ordinated by a 118 Doctor and composed of the Doctor, a professional nurse, two red cross volunteer nurses assisted by 4-6 red cross volunteers was set, in order to help injured people access and handling into the Advanced Medical Centre.

The ambulance continuously shuttled from the First Aid Centre to the Advanced Medical Centre. In order to facilitate and let the transportation faster, the ambulances coming from L'Aquila side continued towards Teramo and vice 
versa. An helicopter ambulance landed on the AMC on the L'Aquila side, took one serious injured guy and delivered him up to "San Salvatore" Civil Hospital, in L'Aquila. Then it returned on the on the L'Aquila side AMC.

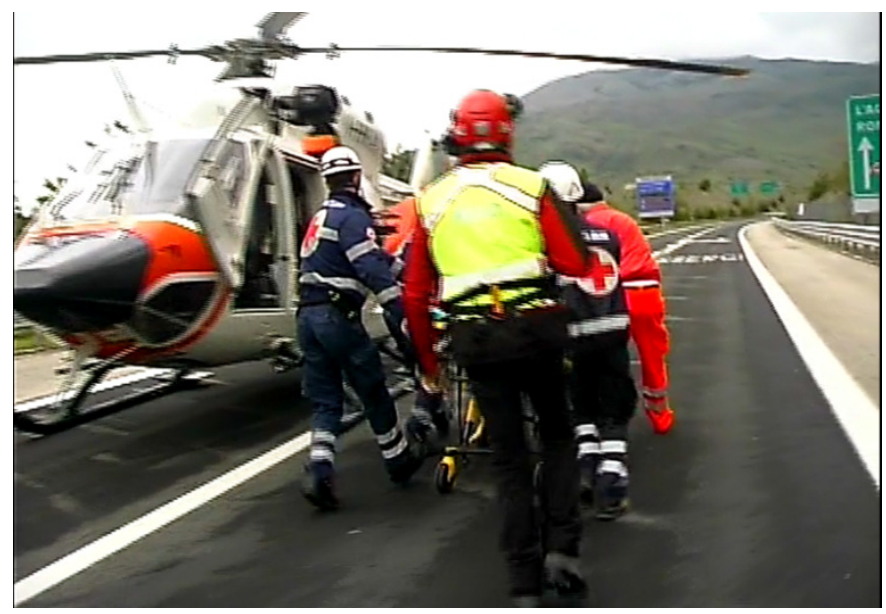

Figure 6: Red Cross volunteer taking the injured guy on the helicopter ambulance.

Once completed the rescue operation and procedures, and verified that the visibility in the tunnel had improved, the Coordination and Command Centre (Fire Brigades) gave the order to proceed with the underground laboratory personnel evacuation. Accordingly with the general emergency plan, the evacuation had to be performed on foot, from the underground labs up to the bypass number 13 , at about $150 \mathrm{~m}$ from the main assembly point inside the laboratories.

One LNGS fireman and a guard, together with the Fire Brigades personnel, using portable lamps, took all the personnel to be evacuated on the other motorway tunnel (the right tunnel), where the 118 vehicles (minivan and ambulances) picked them up and transported them to the tunnel exit. Once completed the evacuation procedure, the vehicles involved in the accident had been removed.

At the end of the crew drill, all the involved entities and personnel reached the LNGS external offices, in Assergi, in order to give a first evaluation and a first opinion exchange about the training test.

\section{Emergency drill results}

Soon after the drill, a meeting has been held in the Majorana Hall of the LNGS external facilities, in order to evaluate the training test results. About 120 people were present at the meeting. All the entities that took part to the crew drill were 
present: L'Aquila Prefecture, organizer of the training test, was quite satisfied about the training outcome, about the personnel behaviour, the results obtained and about the resulting suggestions. One of the most important aspects underlined was that drills are important and they are not an "play act". Their meaning is to highlight any deficiencies, both structural and managerial.

The results can be classified into three main items:

- communication between the entities involved;

- coordination between the entities involved;

- intervention time.

Communication has been the critical point of the drill. The meeting underlined the lack of efficiency of the current equipment and also the lack of communication between the different parts. This led to avoidable errors (i.e. ambulances entering in the wrong direction) and to a poor coordination between rescue teams of the different entities. The problem of communication had been reported since Gran Sasso 2000 drill.

As regards the coordination, the need of the involvement of the Aqueduct has been reported in the meeting. Moreover, a good point would be the identification of a unique referent, let's say a "co-ordinator", for each entity. From a technical point of view, it was highlighted the need to have carriageable bypasses. This can ensure a faster pathway for the rescue vehicles during the emergency.

The intervention time were quite realistic, thanks to the previous organisation of the drill. However, all the entities highlighted an excessive participation of human resources, comparing it to a real situation. A possible suggestion for the next drill has been to involve the "normal operating number of human resources" as people acting in the drill and a limited and well defined number of observers. Moreover, the need of more suitable means and equipments to manage emergencies of this magnitude has been pointed out. By now, the increase of traffic concentration in L'Aquila and Teramo determines the need of a revision of the theoretical time necessary to reach the accident scenario by the rescue teams.

\section{Conclusions}

We can say that the current existing emergency plan has successfully overcome the test related to a "real practice"; notwithstanding this, it has been clearly emphasized that some improvements are needed both as regards the logistic and organizational phase. Furthermore, communication is a key-point to be upgraded; an additional suggestion pointed out was the needed availability of the rescue vehicles.

Another central element in the assessments is the need to involve both the aqueducts (managed by Ruzzo Reti S.p.A. on the Teramo side and by Gran Sasso Acqua S.p.A. on the L'Aquila side) in the organization and implementation process of the emergency drill. This represents a fundamental and central point to consider for the future drills, due to the importance of protecting the water resource reservoir. 
At last but not the least, from the training practice emerged the need of electing an operative working group able to carry out the integration of the suggestions and recommendations and, furthermore, to realize the upgrade of those plants considered of highest priority.

The wide satisfaction for the obtained results should not induce to reduce care and attention to the subject: the General Emergency Plan (PEE) must be subjected to periodical and continuous audits, revisions and tests, in order to maintain the efficiency at the maximum possible level.

\section{References}

[1] LNGS, Rapporto di sicurezza, 2011

[2] LNGS, Piano di Emergenza Interno rev. 03, 2009

[3] Prefettura dell'Aquila, Piano d'Emergenza Esterno (PEE) dei Laboratori Nazionali del Gran Sasso - Laboratori Sotterranei, 2008

[4] ANAS, Gran Sasso - Il traforo autostradale, COGEFAR BASTOGI IRBS, May 1980 